\title{
An Asynchronous Fixed Priority Arbiter for High througput Time Correlated Single Photon Counting Systems
}

\author{
Timothé Turko ${ }^{1}$, Wilfried Uhring ${ }^{1}$, Foudil Dadouche ${ }^{1}$, Laurent Fesquet ${ }^{2}$ \\ 1) ICube, UMR 7357, Université de Strasbourg and CNRS, 23, rue du Loess, 67037 Strasbourg, France \\ 2) TIMA Laboratory, UMR 5159, Université de Grenoble Alpes, 46 avenue Félix Viallet, 38031 Grenoble, France \\ Email: wilfried.uhring@unistra.fr
}

\begin{abstract}
This paper presents a Fixed Priority Arbiter (FPA) which fully takes benefice of asynchronous design. It signs up in a Time Correlated Single Photon Counting system where the data transfer from the Time to Digital Converters to the readout or process unit is a critical aspect. In order to increase the photon counting rate of the setup, the device has to handle a large amount of data following the increasing number of sensors. Thanks to its asynchronous design, the proposed FPA can be easily assembled to build a wide data path tree to guide all the data to a single output. The implementation on a $180 \mathrm{~nm}$ CMOS technology shows that a 128 input wide path tree can achieve a burst input rate of 21 Giga event per second while sustaining an output data rate of 333 Mega event per second.
\end{abstract}

Keywords-Asynchronous circuit, Priority Arbiter, Data selection, Time Correlated Single Photon Counting, Single Photon Avalanche Diode, Time to Digital Converter.

\section{INTRODUCTION}

Nowadays, with the emergence of Single Photon Avalanche Diode (SPAD) fabricated in low-cost standard CMOS technologies, more and more applications based on Time correlated Single Photon Counting (TCSPC) are developed [1]. Those devices measure a precise time duration separating the emission and the detection of a photon thanks to Time to Digital Converters (TDC). However, the association SPAD plus TDC have some performance limitations, especially the SPAD which are limited by their maximal counting rate. Indeed, one elegant solution to increase the maximum counting rate has been proposed in [2] and [3].
Instead of focalizing the light signal on one SPAD, it is possible to split the signal on several SPAD disposed in row or matrix [2] as described on Fig. 1. Each SPAD is associated with a dedicated TDC or, alternatively, several SPAD can share a single TDC [2] or a group of TDC [4]. By splitting the light signal on a large number of sensors, each individual SPAD can operate without reaching its own maximal counting rate which is generally limited to less than 10 Mega photon per second $(\mathrm{ph} / \mathrm{s})$. This results in globally increasing the system counting rate. The drawback of such an architecture, in addition to a larger silicon area, is the data explosion. As many events can be detected by the SPAD system at the same time, collecting and analyzing them is certainly more challenging.

In order to only deliver one single data to the processing unit, an arbitrating unit is mandatory. In this article, an asynchronous Fixed Priority Arbiter (FPA), matching the operating criteria of our SPAD system, is presented. For our purpose, an FPA with a high throughput, able to address each couple SPAD plus TDC, is needed. Asynchronous electronics is the perfect candidate for this kind of specification because it offers reliability, speed, scalability and low power consumption.

The paper is organized as follows: after a brief introduction of the asynchronous logic in the second section, we present the Fixed Priority Arbiter proposed in this work in section III. Section IV shows the simulation results obtained for a 2-stages FPA prototype extensible to an arbitrary size. Finally, a summary of this work will be given in section $\mathrm{V}$.

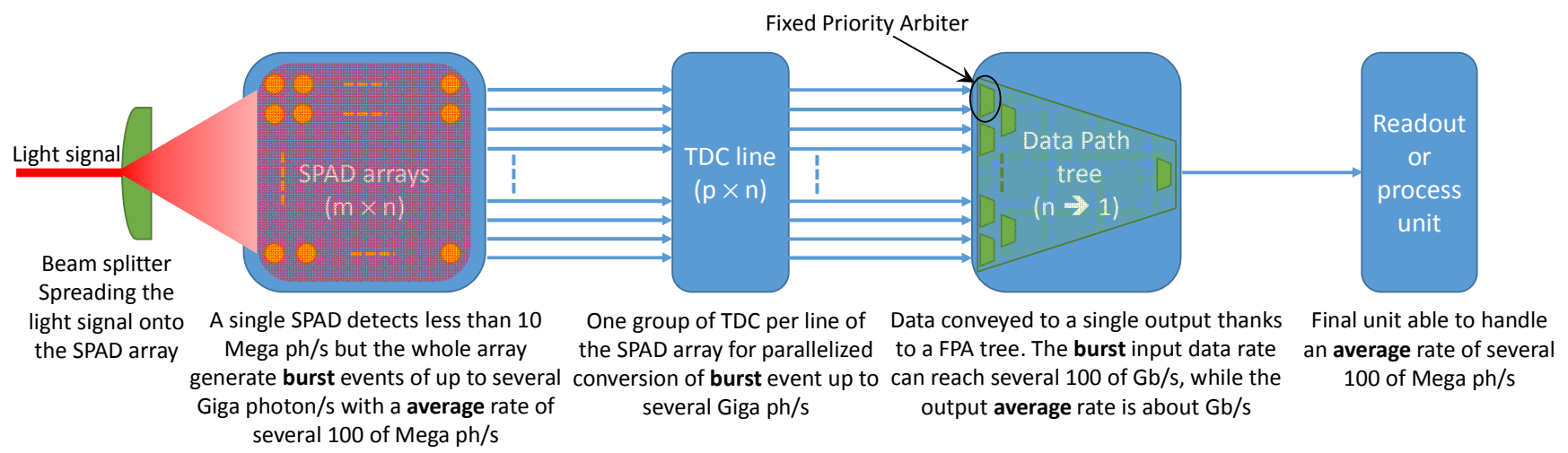

Fig. 1. A high throughput TCSPC system with parallelized photon detection. The light signal is spread on several SPAD sharing a group of in line TDC. The data path tree allows to convey the multiple TDC output data to the readout or process unit 


\section{ASYNCHRONOUS LOGIC}

Nowadays, almost all digital circuits are synchronous. The main characteristic of a synchronous circuit is its global synchronization based on clock signal. Contrarily to clocked systems, the asynchronous circuits are locally synchronized thanks to a handshake protocol. This communication method is able to synchronize complex circuits while guarantying a minimal processing time.

Moreover, asynchronous logic perfectly matches our project needs because, by nature, it is able to reliably manage non-deterministic events [5]. This point is extremely important because sampling with a synchronous system is not reliable, especially if the event number is large and the time between events short, which is exactly our case study! Indeed, due to the quantum nature of the light, it is impossible to predict the time of photon detection by a SPAD. In addition, as asynchronous logic is event-driven, it can react faster than a synchronous system, which is waiting on the clock signal edges to process data.

\section{A. Micro pipeline circuit}

It exists several classes of asynchronous circuits but one of the most popular is the micropipeline class [6]. The latter is really much looking as a synchronous circuit except that there is no global synchronization. Indeed, the clock tree is replaced by a set of small controllers guarantying a perfect synchronization between the circuit stages thanks to a handshake protocol. As an example, the Fig. 2 shows the architecture of a micropipeline asynchronous FIFO. The data propagate through the FIFO thanks to the handshake signals (Req and Ack) that follow a communication protocol. The protocol is implemented in small control units, which embed a few logic and C-elements. C-elements implement a rendez-vous function, i.e. the output takes the same value as the input values are equal and keeps its output value otherwise. In the sequel, the C-element symbol looks like a AND gate with a C inside.

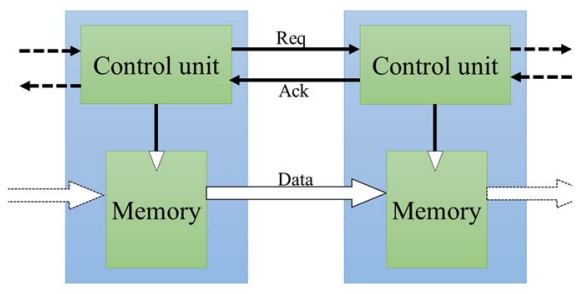

Fig. 2. Micro pipeline asynchronous FIFO

\section{B. Return-to-zero handshake protocol}

As previously explained, asynchronous logic is not synchronized by a clock signal. In order to ensure a correct local synchronization, a communication protocol is implemented. It exists many protocols extensively described in the scientific literature including2-phase and 4-phase protocols [7]. In our design, we used the "Return to Zero handshake" (or 4-phase) protocol, which is a common protocol in asynchronous logic because it allows a small and simple implementation of the handshake controllers (2-phase protocols are more complex).

The Return-to-Zero protocol [8] (see Fig. 3) starts with a request send by the sender block. This emitter also provides at the same time data to the receiver block. The controller of the receiver can start processing data. Once the processing is complete, the receiver acknowledges the emitter block by setting the Acknowledge signal at zero. Then the emitter resets its request, and the return to zero of the request makes possible the reinitialization of the Acknowledge signal to one. This ends the data transaction between the sender and the receiver blocks.

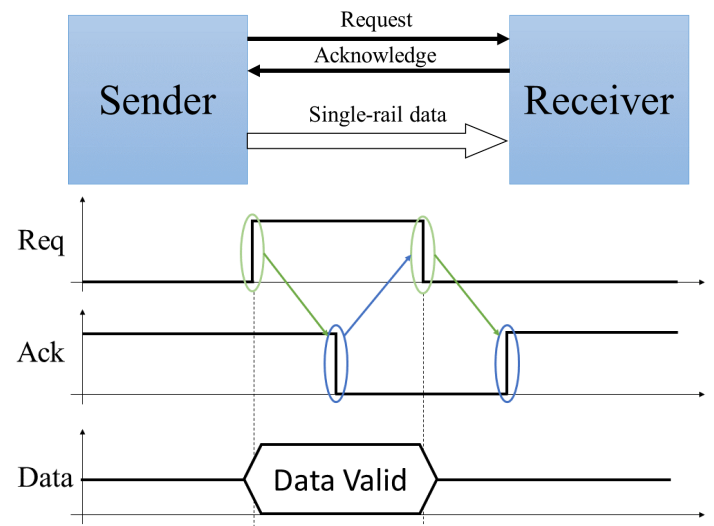

Fig. 3. Functioning principle of the Return-to-zero handshake protocol

\section{FIXED PRIOTITY ARBITER}

The primary function of a Fixed Priority Arbiter is to send to the output only one of several inputs arriving at the same time (or at instants that cannot be distinguished) with a predefined priority given to each input. Indeed, if an event occurs at one of the FPA inputs, the arbiter will put it in competition with the other arriving events or already arrived. Then the PFA will send each event number to the output according to its priority until all the events have been processed. Fixed Priority Arbiters always prioritize the same inputs while Dynamic Priority Arbiters (DPA) [9] are able to dynamically change the priorities. These latter suffer from a lower speed and a more complex implementation which is very penalizing for our application.

In the following, after an introduction of the functioning principle of basic FPA 2- input arbiter, we present the implementation of an elementary cell of the proposed FPA, which will be then instantiated in a based data path tree suitable for TCPC devices. Finally, the addressing mechanism of data position is introduced.

\section{A. 2-input Arbiter functioning principle}

The basic FPA is the 2- input arbiter (cf. Fig. 4). Both input signals are in competition to be granted by the arbiter. If only one request is present on the inputs, the FPA send data to its output. This means that the request propagates through the arbiter as well as the data and the sender block is acknowledged. 


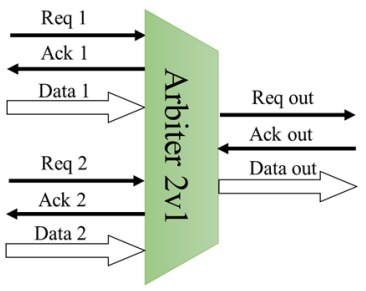

Fig. 4. Arbiter 2 to 1

If both request signals simultaneously occur, only one signal input is granted by the arbiter according to its priority policy. In the sequel, the proposed FPA will always grant first the channel (Req 1, Ack 1, Data 1) as it is shown on Fig. 5.

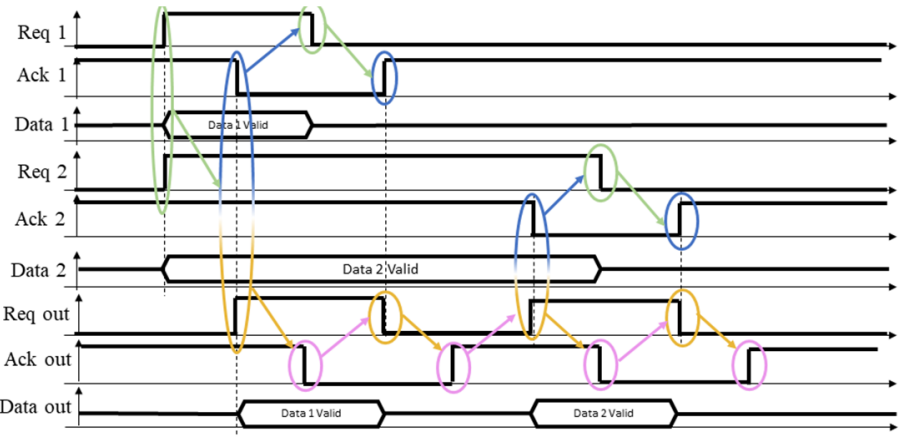

Fig. 5. Functioning principle of the arbiter 2 to 1

When both request occur at the same moment, the FPA grants the first signal input to propagate to the output. Once the next block acknowledges (with the Ack out signal), the second signal input can be granted. As Req_out propagates to the next component, notice that the previous blocks are acknowledged with the signals $A c k 1$ or $A c k 2$.

\section{B. 2-Input Arbiter Implementation}

The proposed Fixed Priority Arbiter with two input channels and one output channel is presented on Fig. 6. As shown in Figure 2, the micropipeline architecture separates the control and data paths. At the entrance of the arbiter, there is three asymmetrical Muller gates [11], one for each request possible case (no need to check the case where both request signals are low because there is no request!). When requests occur, only one of the asymmetrical C-elements is set to one (the conditions are mutually exclusive) and only one data signal is selected by the multiplexer to be send on the output. Then the capture of a new request is locked until the 4-phase transaction is completed as described in Fig. 3.

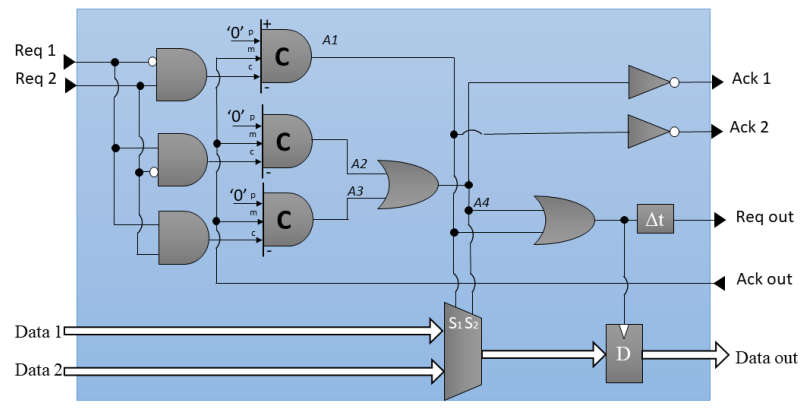

Fig. 6. FPA 2 to 1 , schematic
The transistor level schematic is shown figure 7. The "m" input is always set to " 0 " in order to control the gate output when a request occure on the " $\mathrm{p}$ " input and reset each $\mathrm{C}$ element with an acknowledge signal.

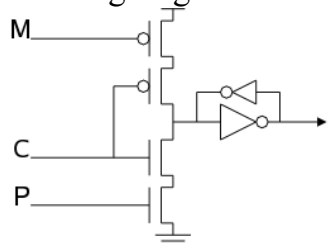

Fig. 7. transistor level design : Asymetrical muller gate

In order to guaranty the local circuit timing assumptions, micropipeline circuits sample the data path signals when the data in the combinational logic are stabilized, i.e. after a time at least corresponding to the local critical path. This is implemented thanks to the delay $\Delta \mathrm{t}$ (see Fig. 6). Therefore a static timing analysis is performed in order to determine the delay value. That way ensures the correct circuit behavior.

\section{Arbiter based data path tree}

In order to design a FPA for our TCSPC device, a large amount of inputs are required. To increase the number of inputs, it is possible to cascade several 2-to-1 FPA. In this manner, a very large number of entries (cf. Fig. 8) can be processed. The last stage of the arbiter tree is connected to the readout of the processing unit.

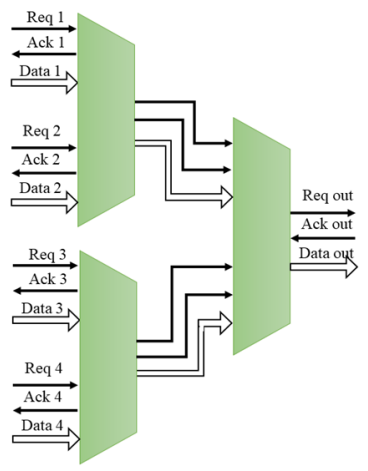

Fig. 8. Arbiter tree structure

In our system, the TDC is the component generating requests, one per photon. The FPA allows the TDC and so the SPAD to perform a new measure once the first stage has acknowledged. The FPA is thus acting as a buffer memory that release the TDC unit as soon as possible. It has been demonstrated in [3] that this feature drastically reduces the risk to not be able to detect the next photon due to the increased dead time that results from the TDC data readout

Then, each measurement data goes through the FPA whatever the arbiter size. Each stage concurrently processes the data in order to determine which one is allowed to be granted first. The local concurrent communications is the real strength of the asynchronous implementation because when a data is in the FPA tree, it will not monopolize the whole path until it reaches the output of the tree. Indeed, each 2-to-1 FPA only exchanges information with its upper and lower stages meaning that the other 2-to-1 FPA are available to move data forward. 
Much information can simultaneously evolve in the FPA chain and they are only temporary blocked when it meet another data. As the stage number is the binary logarithm of the number of input, that the FPA latency tends to evolve logarithmically. This is also depending on the events themselves and on the number of conflicts which are solved in the FPA. Finally, the worst case is obtained when the data of the lowest priority meet a conflict on each 2-to-1 FPA. Thanks to its asynchronous nature, the FPA helps to minimize the data extraction time and so, to maximize the photon counting rate of the TCSPC device.

\section{Addressing the data position}

At this point of our development, the FPA only propagates the data to the output of the tree and the origin of the data is lost. In some TCSPC applications, the position of the photon on the SPAD structure is required. It is possible to include the position of the SPAD directly after the TDC data, by giving an arbitrary number to each SPAD, but in the case where a lot of SPAD is needed, the memory size in the FPA will increase. In order to minimize the silicon area, another addressing method is proposed. At each passage in a 2-to-1 FPA, one bit is added to the data to encode the origin of the request. " 0 " if the origin is the request 1 and " 1 ' if the origin is request 2 . This means that each path is encoded and it's possible to determine the whole data path with a minimal amount of bits. Fig. 9 describes the implementation of this addressing method, where after a Request 1, the 2-to-1 FPA add a " 0 " to the data to symbolized data origins.

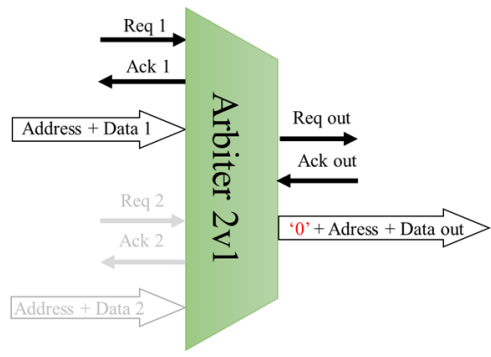

Fig. 9. Example of addressing

The drawback of this address encoding is that each arbiter stage is slightly different from the previous one. Each local memory from the stage $\mathrm{N}+1$ has to instantiate a memory with one additional bit. The size of the memory will increase stage by stage until the final stage.

\section{SIMULATION RESUlTS}

The FPA tree has been implemented in a standard $180 \mathrm{~nm}$ CMOS technology. The worst case simulation has been made to ensure that all the local timing assumptions are met with the implemented delays. Notice that micropipeline circuits are considered more robust to Process, Voltage and Temperature variations than the synchronous circuits.

Simulation is made on 2-stage FPA (4 inputs and 1 output). In order to assess the FPA tree functionality and throughput, 4 request occurred at the same time are generated and then propagate, one after the other, through the FPAs. It takes about $12 \mathrm{~ns}$ to handle the 4 request, i.e. $3 \mathrm{~ns}$ per request to process.
This means that the average readout data rate reach $333 \mathrm{Mevent} / \mathrm{s}$.

By extrapolating the simulation results, it is clear that the presented architecture can be expanded to a larger FPA tree. In our application, the targeted size is 128 inputs. In this case, the first stage possessed 64 memories, thus the maximal achievable burst input rate is $64 \times 333=21$ Gevents per seconds, and the maximal readout rate is still $333 \mathrm{Mevent} / \mathrm{s}$.

\section{CONCLUSION}

A scalable asynchronous fixed priority arbiter has been presented, designed and simulated in a $180 \mathrm{~nm}$ CMOS technology. The use of an asynchronous architecture ensure a high speed as well as an easy scalability. Indeed, by cascading 2-to-1 FPA, a FPA tree can be designed in order to implement a TCSPC system where the data transfer from the TDC to the processing unit is a critical aspect. The system can intrinsically be expanded to an arbitrary size and can achieve a burst input rate of 21 Giga event per second for a 128-input wide tree while maintaining the output rate at 333 Mega event per second.

\section{REFERENCES}

[1] F. Villa, R. Lussana, D. Portaluppi, A. Tosi, F. Zappa, "Time-resolved CMOS SPAD arrays: architectures, applications and perspectives," Proc. SPIE 10212, 102120J, 2017, doi: 10.1117/12.2262586

[2] [Henderson] T. Al abbas, N. Dutton, O. Almer, N. Finlayson, F. Mattioli Della Rocca, R. Henderson, "A CMOS SPAD Sensor with a Multi-Event Folded Flash Time-to-Digital Converter for Ultra-fast Optical Transient Capture.” IEEE Sensors Journal. 2018, Vol. 18, No. 8. pp. 3163-3173, doi: 10.1109/JSEN.2018.2803087

[3] I. Malass, W. Uhring, J-P. Le Normand, N. Dumas, F. Dadouche, "Parallelized Integrated Time Correlated Photon Counting System for High photon counting rate applications," Photon Counting, Chap. 9, pp. 209-224, InTech, 2018, doi:10.5772/intechopen.72273

[4] S. Lindner, C. Zhang, I. M. Antolovic, M. Wolf and E. Charbon, "A 252 x 144 SPAD pixel FLASH LiDAR with 1728 Dual-clock 48.8 ps TDCs, Integrated Histogramming and 14.9-to-1 compression in 180nm CMOS Technology" in 2018 Symposiumon VLSI Circuits, June 2018.

[5] J.-B. Rigaud, J. Quartana, L. Fesquet, M. Renaudin, "High-Level Modeling and Design of Asynchronous Arbiters for On-Chip Communication Systems", DATE, 4-7 March, 2002, Paris, France.

[6] I. E. Sutherland, "Micropipelines, "Communications of the ACM, Vol. 32, pp.720-738, 1989, doi. 10.1145/63526.63532

[7] Principles of Asynchronous Circuit Design. A Systems Perspective. Editors: Sparso, Jens, Furber, Steve (Eds), Springer 2001, ISBN 978-14757-3385-3

[8] E. G. Jung, B. S. Choi, Y. G. Won and D. I. Lee, "Handshake protocol using return-to-zero data encoding for high performance asynchronous bus," in IEE Proceedings - Computers and Digital Techniques, vol. 150, no. 4, pp. 245-251, 18 July 2003. doi: 10.1049/ip-cdt:20030690

[9] Y. Yang, R. Wu, L. Zhang and D. Zhou, "An Asynchronous Adaptive Priority Round-Robin Arbiter Based on Four-Phase Dual-rail Protocol," in Chinese Journal of Electronics, vol. 24, no. 1, pp. 1-7, 01 2015. doi: 10.1049/cje.2015.01.001

[10] J. Simatic, A. Cherkaoui, F. Bertrand, R. P. Bastos and L. Fesquet, "A Practical Framework for Specification, Verification, and Design of SelfTimed Pipelines," 2017 23rd IEEE International Symposium on Asynchronous Circuits and Systems (ASYNC), San Diego, CA, 2017, pp. 65-72. doi: 10.1109/ASYNC.2017.16 